\title{
Impact of Oral Health on Nutritional Status in Community-dwelling Older Adults in Turkey
}

\author{
(1) Sibel Akın, (1) Servet Kesim, (1) Taha Yaşar Manav, (1) Elif Şafak Deniz, (1) Ahmet Öztürk, (1) Mümtaz Mazıcıoğlu, \\ (D) Fırat Özer Firuzan \\ Erciyes University Faculty of Medicine, Department of Geriatrics, Kayseri, Turkey
}

\begin{abstract}
Objective: To determine the association between oral health and nutritional status in community-dwelling older adults in Turkey. A populationbased cohort study using data obtained from the Kayseri Elderly. Health Study.

Materials and Methods: Nutritional status was assessed using the Mini Nutritional Assessment. In oral examination, number of decayed permanent teeth, use of dental prostheses, number of natural teeth, use of toothpaste and tooth brushing frequency were noted. Oral health-related quality of life and periodontal status were assessed by the Oral Health Impact Profile (OHIP-14) questionnaire and the Community Periodontal Index, respectively.

Results: A total of 476 older adults, whose oral health data were available, were included in this study. The mean age of the participants was $71.8 \pm 5.6$ years and $52.3 \%$ were female. The prevalence rate for normal nutritional status was $52.2 \%$. The remaining $44.2 \%$ of the older adults were determined to be at risk of malnutrition and 3.6\% had malnutrition. In multivariate analysis, depressive mood (OR: 2.54 95\% Cl: 1.59-4.06), use of toothpaste (OR: 0.58 95\% Cl: 0.38-0.88) and OHIP scores (OR: 1.03 95\% Cl: 1.01-1.06) were detected to be independent determinants of nutritional status.
\end{abstract}

Conclusion: Depressive mood, not using toothpaste and high OHIP scores were found to be significant risk factors for malnutrition.

Keywords: Older adults, community-dwelling, malnutrition, oral health status, mini nutritional assessment

\section{Introduction}

Malnutrition is frequent in older individuals, and has particular relevance in individuals with the advancing age. Studies have shown that the prevalence of malnutrition is approximately $2-8 \%$ in the community-dwelling older adults (1). The reported prevalence of malnutrition in the community-dwelling Turkish older adult population is in a broad range of 3.3 to $19 \%(2$, 3). Malnutrition is one of the most relevant conditions that negatively affects the health of the older adults. Decline in nutritional quality is one of the complications of old aging (4). Poor oral health, comorbidities, use of medication, social, psychological and cultural factors are other contributing factors to the poor nutritional status of the older adults (5). Oral health problems related with teeth loss, lack of or inadequate prosthesis, the presence of pain related to caries and periodontal diseases are fundamental health problems which may have a high impact on nutrition, primarily in the older adults. These oral health-related problems may cause consumption of a diet low in fiber, and difficulty in fragmentation and grinding of food in the mouth. As a result, decreasing amount of fruit, vegetable, meat and bean consumption in diet may lead primarily increase in carbohydrate and sugar consumption (6). A relatively small number of studies have evaluated whether oral health problems are predictors of nutritional status among community-dwelling older adults by using measurements that assess the risk of malnutrition, especially assessment of malnutrition with Mini-Nutritional Assessment (MNA). The assessment of malnutrition can be done with anthropometric measurements or indexes' derived from these measures and with various scales. The most frequently used

Address for Correspondence: Sibel Akın MD, Erciyes University Faculty of Medicine, Department of Geriatrics, Kayseri, Turkey E-mail: sibelyanmaz@gmail.com ORCID: orcid.org/0000-0002-6139-7254

Received: May 13, 2019 Accepted: Jun 27, 2019

Cite this article as: Akın S, Kesim S, Manav TY, Şafak Deniz E, Öztürk A, Mazıcıoğlu M, Firuzan FÖ. Impact of Oral Health on Nutritional Status in Communitydwelling Older Adults in Turkey. Eur J Geriatr Gerontol 2019;1(1):29-35

๑Copyright 2019 by the Academic Geriatrics Society / European Journal of Geriatrics and Gerontology published by Galenos Publishing House. 
scale to assess nutritional status is the MNA. It is a noninvasive, well-validated, and simple test which is recommended for nutritional assessment in the older adults (1).

To our knowledge, there are studies about the oral health status of the elderly but studies that focus on the association between oral health and nutritional status in the older adults in Turkey are limited. Thus, the aim of this study is both to describe oral health status with reliable measures and to determine whether poor oral status is a contributing factor in the development of malnutrition in the community-dwelling older adults in Turkey.

\section{Materials and Methods}

To assess the relationship between oral health and nutritional status in the community dwelling older adults we used the Kayseri Elderly Health Study (KEHES) data (2). This is a crosssectional population-based study that was conducted from August 2013 to December 2013. Number of older adults in this study constitutes at least 1\% of the community-dwelling older adults in the Kayseri (a city in Central Anatolia in Turkey, with an estimated total population of 1.400.000). Data were collected from 21 Family Health Care Centers (FHCC). The distribution of health centers included in the study was stratified according to socio-economic level; low, moderate and good with respect to socio-economic status in general population. Family physicians in each FHCC was requested to randomly invite six older adults (three male and three female) from each age group who were 65-74, 75-84 and older than 85 years.

Nutritional status was assessed by the Turkish version of MNA long form (7). The MNA is developed by Guigoz et al. (8), which is the most established, best validated and widespread nutritional assessment tool used in geriatric population. Based on the total score, MNA scores of the subjects were classified into three categories. A score of less than 17 points (out of total 30) is regarded as malnutrition, 17-23.5 points at risk for malnutrition and $>23.5$ points at normal nutritional status. Geriatric Depression scale (GDS) (9) and Mini-mental State Examination (MMSE) (10) scores were obtained to screen depression and cognition respectively.

The GDS cut-off point was 14 for Turkish older adults (11) and cognitive impairment was defined as an MMSE score less than 24/30 in the illiterate and 25/30 in the literate individuals (12). Length of education was assessed according to the last school graduated. The level of education was grouped as illiterate, literate ( $<1$ years), $\geq 1-8$ years and $>8$ years (Illiterate, primary, secondary and over). Income was grouped as good, moderate, and low according to self-report of older adults, since income level is considered as a range of perception rather than a quantity.
All dental examinations were performed by an experienced dentist. The oral health status assessment compromised of the number of decayed, missing and filled teeth; periodontal status, Community Periodontal Index (CPI), and use of dental prosthesis (None, fixed, removable).

Subjective oral health-related quality of life was assessed by oral health impact profile (OHIP-14), which was validated for Turkish population (13). This is a 14-item questionnaire that includes seven dimensions and detects an individual's perception of functional limitation, physical pain, psychological discomfort, physical disability, psychological disability, social disability, and handicap. Subjects are asked if they have always/very often, often, sometimes, seldom or never experienced any of those problems in the previous three months. Responses are scored on a scale ranging from 1 to 5 (14). Higher scores indicate worse oral health-related quality of life.

Periodontal status was assessed by the CPI as it is recommended by World Health Organization. The null CPI score corresponds to healthy periodontal condition. Increasing CPI scores, according to the severity of periodontal status, are described as 1: gingival bleeding; 2 : calculus and bleeding; 3 : shallow periodontal pockets (Pockets 4-5 $\mathrm{mm}$ ); 4: deep periodontal pockets (6 millimeters or more) (15). CPI score 1 corresponds to periodontal disease which can be improved with domestic oral hygiene; a score of 2 or 3 needs intervention by a dentist. The most severe condition, $\mathrm{CPI} 4$, means that an individual requires additional periodontal surgery. Participants were categorized according to dentures: none, fixed and removable dentures. The entire study protocol was approved by the Medical Ethics Committee of Erciyes University Medical Faculty (No: 2013/441). Participants without severe cognitive impairment and who gave their informed consent were recruited; for participants with mild or moderate cognitive impairment, consent was obtained from a proxy.

\section{Statistics}

Descriptive characteristics of demography, cognitive status and depressive symptoms of community dwelling older adults were determined. These parameters were compared in our study group according to the nutritional status by chi-square test. Another comparison between normal nutritional status and poor nutrition (malnutrition/malnutrition risk) were done for oral health status; number of natural teeth, frequency of tooth brushing, decayed teeth, periodontal status, use of dental prosthesis, and oral health-related quality of life. Each parameter related with nutritional status then analyzed with logistic regression as uni-, and multivariate dependent variable. $\mathrm{P}<0.05$ value was considered statistically significant. Data were analyzed by IBM $^{\odot}$ SPSS ${ }^{\odot}$ Statistics version 22 (IBM ${ }^{\odot}$ Corp., Armonk, NY, USA) 


\section{Results}

We included 468 community-dwelling older adults with a mean age of $71.8\left(S S_{ \pm} .6\right)$ years and their age range was 60 to 91years. Of these $52.3 \%$ were females and $47.7 \%$ were males. We found that $52.1 \%(n=244)$ of subjects were at normal nutritional status, 44.2\% $(n=207)$ had Ministry of Natural Resources (MNR) status and $3.7 \%(n=17)$ had membranous nephropathy (MN) according to Medical Nurse Associate (MNA). Since only 17 of the older participants were classified as malnourished, we combined the MN/MNR groups. The characteristics of the study group according to nutritional status are summarized in Table 1.

When compared normal nutritional status and MN/MNR subjects by sex, MN/MNR was prevalent in the female gender. Age was a significant indicator of being poor or normal nutritional status, $72.5\left(\mathrm{SD}_{ \pm} 5.9\right)$ years for poor and 70.8 $(S D=5.3)$ years for normal nutritional status. The MN/MNR were about two times higher in illiterate than literate older adults and normal nutritional status were two times higher in 1-8 years of educated older adults than literate. The ratio of older adults with cognitive impairment was about two times

\begin{tabular}{|c|c|c|c|c|}
\hline Socio-demographic variables & All n (\%) & $\begin{array}{l}\text { Malnutrition/ Malnutrition risk } \\
\text { n (\%) }\end{array}$ & $\begin{array}{l}\text { Normal nutritional status } \\
\mathrm{n}(\%)\end{array}$ & $\begin{array}{l}p \\
\text { values }\end{array}$ \\
\hline \multicolumn{5}{|l|}{ Age } \\
\hline Mean \pm SD & $71.8(5.6)$ & $72.5(5.9)$ & $70.8(5.3)$ & \multirow[b]{2}{*}{$<0.001$} \\
\hline Age groups & - & - & - & \\
\hline $65-74$ & $320(67.2)$ & $144(64.3)$ & $169(69.3)$ & \multirow{3}{*}{0.721} \\
\hline $75-84$ & $132(27.7)$ & $68(30.4)$ & $63(28.3)$ & \\
\hline$\geq 85$ & $10(2.1)$ & $5(2.2)$ & $5(2.0)$ & \\
\hline \multicolumn{5}{|l|}{ Sex } \\
\hline \multicolumn{5}{|l|}{ Smoking } \\
\hline Yes & $166(34.9)$ & 70 (31.2) & $94(38.5)$ & \multirow{2}{*}{0.099} \\
\hline No & $310(65.1)$ & $154(68.8)$ & $150(61.5)$ & \\
\hline \multicolumn{5}{|l|}{ Education level } \\
\hline Illiterate & $172(36.1)$ & $107(47.8)$ & $62(25.4)$ & \multirow{4}{*}{$<0.001$} \\
\hline Literate & $98(20.6)$ & $45(20.1)$ & $52(21.3)$ & \\
\hline $1-8$ years & $173(36.3)$ & $62(27.7)$ & $107(43.9)$ & \\
\hline$>8$ years & $33(6.9)$ & $10(4.5)$ & $23(9.4)$ & \\
\hline Yes & $124(26.2)$ & 73 (32.6) & $48(19.8)$ & \multirow{2}{*}{0.002} \\
\hline No & 350 (73.8) & $151(67.4)$ & $194(80.2)$ & \\
\hline \multicolumn{5}{|l|}{ Depression } \\
\hline Yes & $138(29.1)$ & $94(42.0)$ & 40 (16.5) & \multirow{2}{*}{$<0.001$} \\
\hline No & 336 (70.9) & $130(58.0)$ & 202 (83.5) & \\
\hline
\end{tabular}


high in MN/MNR. Depressive symptoms were also indicator of malnutrition both in normal and poorly (MN/MNR) nourished older adults. Additionally, we detected the ratio of nondepressive was higher in normal nutritional status compared with poorly nourished (Table 1).

The average number of teeth in the studied sample was 3.2 $(\mathrm{SD}=4.0)$ and $37.4 \%$ of the subjects were edentulous. The edentulous older adults comprised about one fourth of our sample $(n=178)$. In a comparison of the normal nutritional status and MN/MNR older adults, about $1 / 3$ of the normal nutritional status and half of MN/MNR were edentulous. Using toothpaste and brushing teeth rate was about 50\%. MN/MNR was higher in non-toothpaste users (59.4\%) than non-toothbrushers (53.1\%) (Table 2).

The mean score of OHIP was $12.7(\mathrm{SD}=11.8)$ in MN/MNR and $7.1(\mathrm{SD}=7.7)$ for normal nutritional status older adults. Impairment in oral health-related quality of life (increased OHIP score) was at least 50\% high in MN/MNR. Periodontal evaluation was done in 295 non-edentulous older adults. We found that just $8.8 \%$ of them were healthy. The frequency of periodontal pathologies was as follows: bleeding $1.0 \%$, calculus $8.0 \%$ and sulcus $81.2 \%$. We could not find any difference in CPI scores between the MN/MNR and normal nutritional status.

In bivariate analyses, demographical characteristics (gender, educational levels, and income), cognitive impairment, depression, oral health indicators (use of toothpaste, toothbrushing and oral health-related quality of life) were tested. In multivariate analysis among the above-mentioned independent variables only depressive mood (OR: $2.5495 \% \mathrm{Cl}: 1.59-4.06)$, use of toothpaste (OR: 0.58 95\% Cl: 0.38-0.88) and OHIP (OR: 1.03 95\% Cl: 1.01-1.06) were detected as independent determinants of nutritional status (Table 3).

\section{Discussion}

Malnutrition is one of the major geriatric problems associated with functional decline, poor health status and high mortality (4). Oral health status can be considered as a significant parameter that determines the nutritional level. Oral health status determines both the selection and consumption of food particularly in older adults. Certain types of food (difficult to chew and grind) ingestion may be avoided because of decayed, lost teeth and prosthesis (16). These limitations then may lead decrease in intake of products such as meat, fruit,

\begin{tabular}{|c|c|c|c|c|}
\hline Oral health status & \begin{tabular}{|l|l|} 
All \\
$n(\%)$
\end{tabular} & $\begin{array}{l}\text { Malnutrition/Malnutrition risk } \\
\text { n (\%) }\end{array}$ & $\begin{array}{l}\text { Normal nutritional } \\
\text { status } \\
\text { n (\%) }\end{array}$ & $\begin{array}{l}p \\
\text { values }\end{array}$ \\
\hline \multicolumn{5}{|c|}{ Number of natural teeth } \\
\hline Edentulous & $178(37.4)$ & $93(41.5)$ & $80(32.8)$ & \multirow{3}{*}{0.054} \\
\hline $1-9$ & 256 (53.8) & $117(52.2)$ & $137(56.1)$ & \\
\hline$\geq 10$ & $42(8.8)$ & $14(6.2)$ & $27(11.1)$ & \\
\hline \multicolumn{5}{|c|}{ Tooth brushing frequency } \\
\hline No brushing & $218(45.8)$ & $119(53.1)$ & $93(38.1)$ & \multirow{3}{*}{0.005} \\
\hline$\leq$ Once a day & $175(36.8)$ & $70(31.2)$ & $103(42.2)$ & \\
\hline$>$ Once a day & 83 (17.4) & 35 (15.6) & $48(19.7)$ & \\
\hline \multicolumn{5}{|l|}{ Use of toothpaste } \\
\hline Yes & $239(50.2)$ & $91(40.6)$ & $144(59.0)$ & \multirow{2}{*}{$<0.001$} \\
\hline No & $237(49.8)$ & $133(59.4)$ & $100(41.0)$ & \\
\hline \multicolumn{5}{|l|}{ Decay } \\
\hline Yes & $176(37.0)$ & $84(37.5)$ & $89(36.5)$ & \multirow{2}{*}{0.819} \\
\hline No & $300(63.0)$ & $140(62.5)$ & $155(63.5)$ & \\
\hline \multicolumn{5}{|l|}{ Dental Prosthesis } \\
\hline No & $83(17.6)$ & 43 (19.3) & $39(16.2)$ & \multirow{3}{*}{0.077} \\
\hline Fixed prosthesis & $41(8.7)$ & $13(5.8)$ & $28(11.6)$ & \\
\hline Removable prosthesis & $348(73.7)$ & $167(74.9)$ & $174(72.2)$ & \\
\hline OHIP & $9.8(10.2)$ & $12.7(11.8)$ & $7.1(7.7)$ & $<0.001$ \\
\hline \multicolumn{5}{|l|}{ Periodontal status } \\
\hline (CPI scores) & $5.2(5.5)$ & $4.5(5.2)$ & $5.8(5.6)$ & 0.206 \\
\hline
\end{tabular}




\begin{tabular}{|c|c|c|c|c|}
\hline Variables & \multicolumn{2}{|c|}{ Univariate analysis } & \multicolumn{2}{|c|}{ Multivariate analysis } \\
\hline \multicolumn{5}{|l|}{ Sex } \\
\hline Female & 1 & - & - & - \\
\hline \multicolumn{5}{|c|}{ Education level } \\
\hline Illiterate & 1 & - & - & \multirow{3}{*}{-} \\
\hline Literate & $0.56(0.31-0.99)$ & 0.04 & - & \\
\hline $1-8$ years & $0.50(0.28-0.88)$ & 0.01 & - & \\
\hline Moderate & $1.03(0.60-1.79)$ & 0.89 & - & \multirow[t]{2}{*}{-} \\
\hline Low & $1.14(0.61-2.09)$ & 0.67 & - & \\
\hline \multicolumn{5}{|c|}{ Cognitive impairment } \\
\hline No & 1 & - & - & \multirow{2}{*}{-} \\
\hline Yes & $1.25(0.75-2.05)$ & 0.38 & & \\
\hline \multicolumn{5}{|c|}{ Depressive mood } \\
\hline No & 1 & - & & $<0.001$ \\
\hline Yes & $2.47(1.52-4.04)$ & $<0.001$ & $2.54(1.59-4.06)$ & \\
\hline No & 1 & - & $0.58(0.38-0.88)$ & 0.01 \\
\hline Yes & $0.40(0.17-0.94)$ & 0.037 & & \\
\hline OHIP & $1.03(1.01-1.06)$ & 0.002 & $1.03(1.01-1.06)$ & $<0.001$ \\
\hline CPI & $0.98(0.94-1.02)$ & 0.43 & - & - \\
\hline
\end{tabular}

and vegetables. Even low intake of protein may solely give rise to loss of muscle mass and strength that is a significant determinant of sarcopenia. Ruling out limitations leading to food restriction may prevent increased incidence of falls and fractures, functional impairments and disability related with sarcopenia (5). Above-mentioned conditions about the relationship between oral health and nutrition in older adults are not shown in national studies.

In our study, independent of age and gender, we found that $52.1 \%$ of the older adults were classified as normal nutritional status; $44.2 \%$ as MNR and $3.7 \%$ as having malnutrition according to the MNA test. To our knowledge this is the first study that used MNA to assess the relationship between oral health and nutritional status in the community-dwelling older adults in Turkey in a quite big sample.

Although in many studies abroad MNA have been used to assess the nutritional status in the older adults, few of these examined the relation with both teeth and periodontal pathologies. Additionally, in most of these studies subjects were institutionalized older adults. Similar with this study 
our data support the hypothesis that existing number of natural, non-decayed teeth are not determinants of nutritional status $(17,18)$. The finding that more than onethird of older adults in our study was edentulous supports the consideration that oral health status may be related with malnutrition but tremendous number of older adults with worst oral health condition prevented us to make this discrimination. There are several studies indicating that being edentulous and decreased masticatory capacity is related with malnutrition $(19,20)$. Since we did not collected information about masticator and salivation capacity, we cannot make any evaluation about the effects of these characteristics on malnutrition in the older adults. The major discriminative contribution of our study was the recruitment of community-dwelling elderly who were relatively younger compared to similar studies.

Another contribution of our study was using OHIP-14 to reflect older adults view of quality of life related with oral health in the community-dwelling older adults in Turkey. In the current literature, Barrios et al. (21) shows the association of oral health-related quality of life (OHIP-14) and nutritional status in older adults who has oropharyngeal carcinoma. They found that older adults patients with MN or MNR had considerably worse oral health-related quality of life than those with adequate nutrition. Our findings were consistent with the Barrios et al. (21) study indicating that odds of OHIP on MN/MNR was 1.03 when compared with normal nutritional status. Then we may conclude that OHIP can be a tool of discrimination between MN/MNR and normal nutritional status.

The strong correlation between OHIP and Geriatric Oral Health Assessment Index (GOHAl) provides the opportunity of including another tool for older adults for assessment of oral health related nutritional status. The characteristics of OHIP-14 in determining social impact, and GOHAl in physical function show the significant contribution of this study (22).

Although there are several studies about the inverse relationship between body mass index (BMI) and periodontal health, there are very few about the relationship between MNA and periodontal health. Although BMI may be considered as a simple and basic method to assess nutritional and oral health status it has a limitation in older adults because kyphosis is frequent in older adults (23). We consider that the limitation of BMI may be a good reason to use MNA in assessment of periodontal health in the older adults. However, we could not find any difference between MN/MNR and normal nutritional status in terms of periodontal health.

The limitations of our study can be listed as lack of data in the KEHES study about chewing, swallowing functions and type of consumed food (meat, vegetables and grain).

\section{Conclusion}

The relationship between oral health and nutritional status in older adults is well known. According to our findings we may conclude that depressive mood, perception of oral health quality may be the prominent determinants in nutritional status of older adults. Contribution of this study may then be concentrating on relieving depressive state and improving personal oral health care measures may be the most promising clinical approach for older adults.

\section{Ethics}

Ethics Committee Approval: The entire study protocol was approved by the Medical Ethics Committee of Erciyes University Medical Faculty.

Informed Consent: Participants without severe cognitive impairment and who gave their informed consent were recruited; for participants with mild or moderate cognitive impairment, consent was obtained from a proxy.

Peer-review: Externally peer-reviewed.

\section{Authorship Contributions}

Concept: S.A., Design: S.A., M.M., S.K., Data Collection or Processing: S.A., S.K., T.Y.M., E.Ş.D., M.M., F.Ö.F., Analysis or Interpretation: A.Ö., Literature Search: S.A., Writing: S.A.

Conflict of Interest: No conflict of interest was declared by the authors.

Financial Disclosure: The authors declared that this study received no financial support.

\section{References}

1. Guigoz Y. The Mini Nutritional Assessment (MNA) review of the literatureWhat does it tell us? J Nutr Health Aging 2006;10:466-487.

2. Akin S, Safak ED, Coban SA, Mucuk S, Kiris Y, Ozturk A, Mazıcıoğlu M, Göçer S. Nutritional status and related risk factors which may lead to functional decline in community-dwelling Turkish elderly. Eur Geriatr Med 2014;5:294-297.

3. Gündüz $E$, Eskin $F$, Gündüz $M$, Bentli $R$, Zengin $Y$, Dursun $R$, İçer $M$, Durgun HM, Gürbüz H, Ekinci M, Yeşil Y, Güloğlu C. Malnutrition in CommunityDwelling elderly in Turkey: A Multicenter, Cross-Sectional Study. Med Sci Monit 2015;15:2750-2756.

4. Kaiser MJ, Bauer JM, Rämsch $C$, Uter W, Guigoz Y, Cederholm T, Thomas DR, Anthony PS, Charlton KE, Maggio M, Tsai AC, Vellas B, Sieber CC; Mini Nutritional Assessment International Group. Frequency of malnutrition in older adults: a multinational perspective using the mini nutritional assessment. J Am Geriatr Soc 2010;58:1734-1738

5. Brownie $\mathrm{S}$. Why are elderly individuals at risk of nutritional deficiency? Int J Nurs Pract 2006;12:110-118.

6. Savoca MR, Arcury TA, Leng $X$, Chen $H$, Bell RA, Anderson AM, Kohrman T, Frazier RJ, Gilbert GH, Quandt SA. Severe tooth loss in older adults as a key indicator of compromised dietary quality. Public Health Nutr 2010;13:466-474.

7. Sarikaya D, Halil M, Kuyumcu ME, Kiliç MK, Yesil Y. A Validity Study of Long and Short (MNA-SF) Forms of Mini Nutritional Assessment (MNA) Test in Geriatric Patients. Presented in the 7th Academic Geriatrics Congress Abstract, Antalya, Turkey, 2014. 
8. Guigoz Y, Vellas B, Garry PJ. Assessing the nutritional status of the older adult: the mini nutritional assessment as part of the geriatric evaluation. Nutr Rev 2014;54:59-65.

9. Yesavage JA, Brink TL, Rose TL, Lum O, Huang V, Adey M, Leirer VO. Development and validation of a geriatric depression screening scale: a preliminary report. J Psychiatr Res 1982-1983;17:37-49.

10. Folstein MF, Folstein SE, McHugh PR. "Mini-mental state.' A practical method for grading the cognitive state of patients for the clinician. J Psychiatr Res 1975;12:189-198.

11. Ertan T, Eker E. Reliability, validity, and factor structure of the Geriatric Depression Scale in Turkish older adult: are there different factor structures for different cultures? Int Psychogeriatr 2000;12:163-172.

12. Ertan E, Eker E, Gurgen C. The Standardized Mini-mental State Examination for illiterate Turkish older adult populations. Presented at the second International Symposium on Neuropsychological Assessment of Mental and Behavioral Disorders. Abstract, Bursa, Turkey, 1999.

13. Mumcu G, Inanc N, Ergun T, Ikiz K, Gunes M, Islek U, Yavuz S, Sur H, Atalay T, Direskeneli H. Oral health related quality of life is affected by disease activity in Behcet's disease. Oral Dis 2006;12:145-151.

14. Slade GD. Derivation and validation of a short-form oral health impact profile. Community Dent Oral Epidemiol 1997;25:284-290.

15. WHO Scientific Group on Epidemiology, Etiology and Prevention of Periodontal Diseases: WHO Technical Report Series, No. 621 Geneva, World Health Organization; 1978:7-9.
16. Mesas AE, Andrade SM, Cabrera MA, Bueno VL. Oral health status and nutritional deficit in noninstitutionalized older adults in Londrina, Brazil. Rev Bras Epidemiol 2010;13:434-445.

17. Lamy M, Mojon P, Kalykakis G, Legrand R, Butz-Jorgensen E. Oral status and nutrition in the institutionalized older adult. J Dent 1999;27:443-448

18. Griep MI, Mets TF, Collys K, Ponjaert-Kristoffersen I, Massart DL. Risk of malnutrition in retirement homes older adult persons measured by the "Mini-Nutritional Assessment". J Gerontol A Biol Sci Med Sci 2000;55:57-63.

19. Soini $H$, Muurinen $S$, Routasalo $P$, Sandelin $E$, Savikko $N$, Suominen $M$, Ainamo A, Pitkala KH. Oral and nutritional status is the MNA a useful tool for dental clinics. J Nutr Health Aging 2006;10:495-499.

20. Dion N, Cotart JL, Rabilloud M. Correction of nutrition test errors for more accurate quantification of the link between dental health and malnutrition. Nutrition 2007;23:301-307.

21. Barrios R, Tsakos G, Garcia-Medina B, Martínez-Lara I, Bravo M. Oral healthrelated quality of life and malnutrition in patients treated for oral cancer. Support Care Cancer 2014;22:2927-2933

22. El Osta N, Tubert-Jeannin S, Hennequin M, Bou Abboud Naaman N, El Osta L, Geahchan N. Comparison of the OHIP-14 and GOHAI as measures of oral health among older adult in Lebanon. Health Qual Life Outcomes 2012;30:131.

23. Cook Z, Kirk S, Lawrenson S, Sandford S. Use of BMI in the assessment of undernutrition in older subjects: reflecting on practice. Proc Nutr Soc 2005:64:313-317. 